\title{
AISLAM IE NTO DE Leishmania brazilienzis braziliensis en Proechimys sp. CAPTURADO EN EL RIO NAPO, LORETO - PERU
}

Braga V ela Janeth $\left({ }^{*}\right)$

García M artínez Edward $\left(^{* *}\right)$

Viena del A guila M áximo (*)

Braga Ribeiro Roseli $\left({ }^{* * *}\right)$

\section{RESUMEN}

Se reporta el hallazgo de Proechimys sp. procedente del río Napo, provincia de M aynas, departamento de Loreto, Perú, infectado con Leishmania braziliensis braziiensis.

Mediante trampas Tomahawk se capturé roedores silvestres en dos (2) localidades del río $\mathrm{Napo}$; sacrificados los roedores capturados, se obtuvo muestras de vísceras y piel que se inocularon en hamsters, de donde se aislé en cultivos de Agar sangre - Difco.

Por electroforesis en gel de almidón se corrieron diez (10) enzimas, cuyos perfiles fueron comparados con las cepas patrón de L eishmania mexicana amazonensis. L eishmania braziliensis panamensis Leishmania braziliensis guyanensis y Leishmania braziliensis braziliensis. De cuatro especímenes capturados uno resulté positivo a Leishmania en el hígado, al ser identificado resulté ser semejante a Leishmania braziliensis braziliensis.

Se discute la coincidencia con afirmaciones de otros autóres, de que los huespedes naturales de Leishmania brazilienis braziliensis son roedores. A simismo, se analiza la probable presencia de otros tipos de Leishmania en el área.

Esta es la primera comunicación de este tipo en el país.

\section{SUM MARY}

We are reporting Proechimys sp. from río Napo, Prov. M aynas, Department of L oreto, Peru, infected with L eishmania braziliensis braziliensis. 
U sing tomahawk snare we captured wild rodent in two localities of río $\mathrm{N}$ apo, in which we extracted samples of viscera and skin that were inoculated in hamster, then it was isolated in agar.

By electrophorsis in starch Gel, it was run 10 enzymes profiles which were compire with patterns of cepas of Leishmania mexicana Leishmania braziliensis panamensis Leishrnania braziliensis guyanensis, and Leishmania braziliensis braziliensis. Of the four captured specimens, one resulted possitive to Leishmania in the liver which we are discussing the coincident with the affirmation of the other autors that the visitor of L eishmania braziliensis braziliensis are rodent.

This is the first report of this kind of work in Peru.

\section{INTRODUCCION}

El escaso conocimiento de la epidemiología de la L eishmaniasis cutaneomucosa en el departamento de Loreto, ha impulsado a los autores a iniciar su estudio, en búsqueda de probables reservorios de esta enfermedad en animales silvestres, en áreas donde se están efectuando estudios clínico - parasitológicos (1)

El contado permanente del hombre de la amazonía con los animales silvestres, por razones de alimentación y supervivencia, permite suponer que la enfermedad posee un reservorio natural entre ellos, posibilitando la persistencia del ciclo vital de la Leishmania, siendo el hombre afectado accidentalmente al ingresar a las zonas del monte y ponerse en contacto con el vector, que vive normalmente alimentándose de estos animales. (2) En áreas geográficas del alto $\mathrm{N}$ apo, donde se detectaron pacientes de L eishmaniasis, se capturaron animales silvestres mediante la colocación de trampas en el monte. Un ejemplar de Proechimys sp. capturado en la quebrada A ushiri, presentó lesiones compatibles con L eishmaniasis (3), aunque el aislamiento y cultivo de stock de L eishmania se efectuó del hígado y fue caracterizado por el método de Electroforesis de Isoenzimas, en gel de almidón.

El presente trabajo realizado con financiamiento de la Universidad $\mathrm{Nacional}$ de la A mazonía Peruana (UNAP) yen convenio con la Unidad Departamental de Salud de Loreto.

\section{MATERIALY METODOS}

Territorio: Para el estudio se seleccionaron dos localidades en la cuenca del río Napo A ushiri y M azán, (Fig. 1) que reunieron condiciones de accesibilidad y presencia de monte silvestre a 2 horas de caminata.

M étodo de muestreo: En lugares de monte alto, se colocaron 4 trampas para especies vivas, Tomahawk (4), en horas de la tarde, permaneciendo durante toda la noche; a la mañana siguiente, se recogieron los especímenes capturados, repitiéndose este trabajo durante 2 noches seguidas en cada localidad. 


\section{FIGURA 1}

\section{LUGARES DE CAPTURA DE ROEDORES}

RIO NAPO - DPTO. LORETO

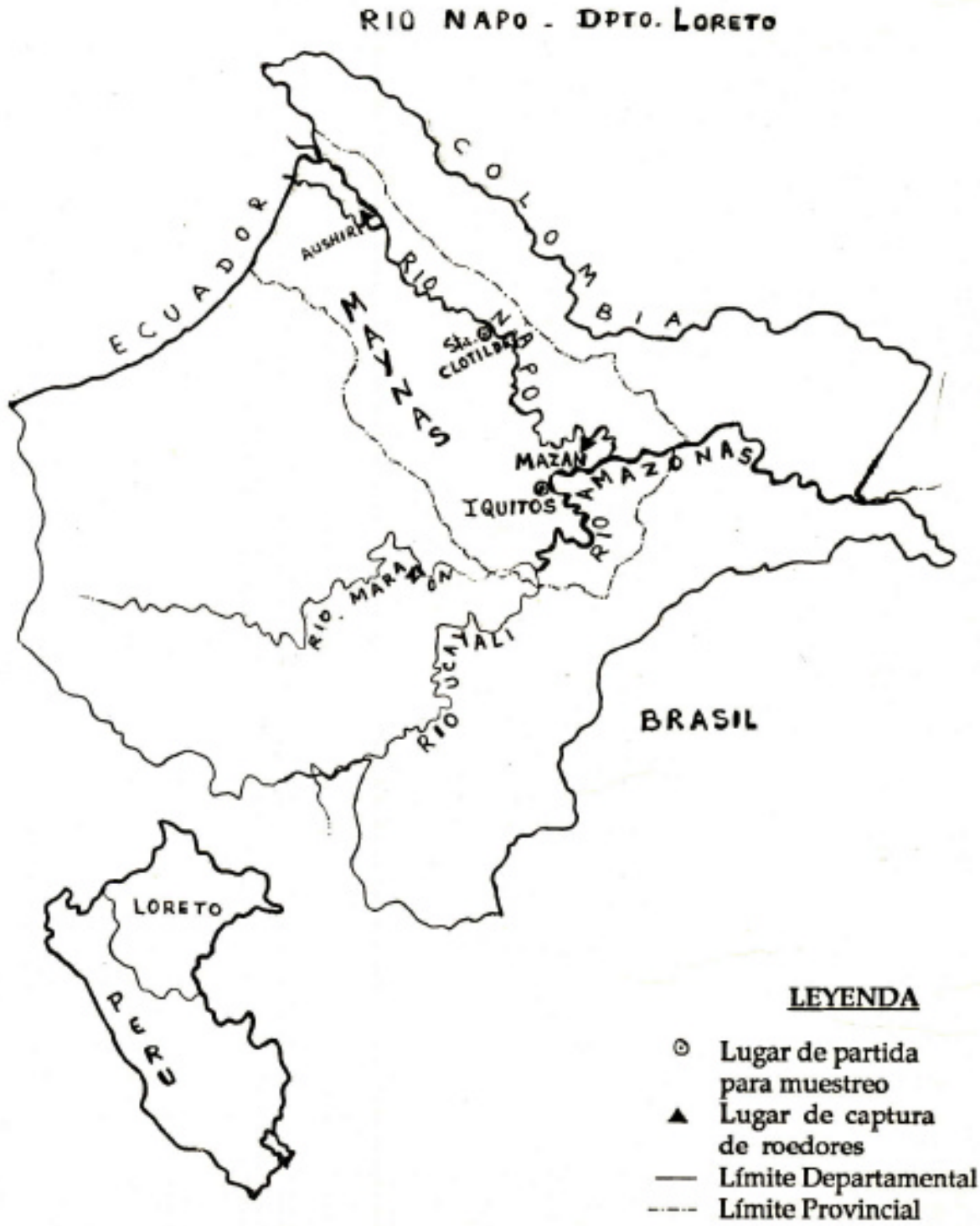


Aislamiento de stock de Leishmania: LoS animales capturados fueron observados durante la semana, para detectar cambios de conducta, y posteriormente trasladados en jaulas metálicas hasta la ciudad de Iquitos, donde eran sacrificados aquellos con lesiones sugerentes de Leishmaniasis o puestos en observación durante 3 meses los que no presentaban sintomatología; al término del tiempo considerado, fueron sacrificados, y se obtuvieron las muestras de piel, hígado y bazo (5). U na parte de esta muestra sirvió para hacer el estudio histopatológico y la otra para homogenizar en suero salino estéril con antibióticos e inocular en patas y hocico del hamster (6), de donde se realizó el aislamiento por microaspirado (7) y se cultivó el material en tubos de cultivo con Agar sangre-Difco-bifásico; según procedimiento descrito por Romero, et al (8). El crecimiento masivo se realizó inoculando en 10 tubos de cultivo con el medio anterior, según procedimiento descrito por Braga R. (9), preparándose el extracto del sobrenadante y conservándolo a 70ํㅡ hasta su utilización.

\section{CARACTERIZACION ISOENZIMATICA}

Mediante el método de Electroforesis en gel de almidón, se estudiaron las siguientes enzimas:
E.C: 2.6 .1 .1 .
E.C: 2.6 .1 .2 .
E.C: 5.3 .1 .9 .
E.C: 1.2 .1 .49
E.C: 2.7 .5 .1 .
E.C: 1.1 .1 .44
E.C: 1.1 .1 .37
E.C: 4.2 .1 .1 .3
E.C: 5.3 .1 .8 .
E.C: 3.4 .11 .1

A spartatoaminotransferasa (ASAT)
A laminoaminotransferasa (ALAT)
Glucosafosfatoisomerasa (GPI)
Glucosa -6- fosfodeshidrogenasa (G-6-PD)
F osfoglucomutasa (PGM)
6-F osfoglucodeshidrogenasa (6 PGDH)
M álico deshidrogenasa (M D H)
A conitasa hidratasa (A CON)
M álico fosfatoisomerasa (M PI
A minopeptidasa (citosol) (PEP)

Las pruebas electroforéticas se realizaron de acuerdo al procedimiento de Miles, donde se detallan los reactivos utilizados y las condiciones de corrida (10). Se utilizaron como cepas la referencia a:

Leishmania braziliensis braziliensis

Leishmania braziliensis guyanensis

Leishmania braziliensis panamensis

L eishmania mexicana amazonensis

Los datos del stock de Leishmania son:
Código

M AN/BR/1975/M 22903

MAN/BR/1975/M 4147

MAN/PA/1975/M 4037

VEC/BR/1967/PHB
H uésped

Hombre L.M.C. Pará-Brasil

Hombre L.C.

Hombre L.C.

Lutzomvia

Flaviscutellata

Procedencia

Pará - Brasil

Panamá

Pará - Brasil

Roedor L.V.
NapoL oreto Perú 


\section{INDICE DE SIMILARIDAD}

Se determiné el índice de similaridad para el stock caracterizado, comparándolo con la presencia o ausencia del carácter isoenzimático en los patrones de las cepas de referencia utilizadas, de acuerdo a procedimientos descritos por Réady (11) y Godfrey (12). L a ecuación de referencia es la siguiente:
IS. $=\frac{z}{a+b-z}$
I.S. = Indice de similaridad
$a=\# \quad$ de bandas de stock (a)
$b=\# \quad$ de bandas de stock (b)
$\mathrm{c}=\# \quad$ de bandas compartidas

\section{RESULTADOS}

Se capturaron 4 especímenes de roedores: tres de ellos en la localidad de Mazán, los que fueron 2 "ratas de campo. Rattus rattus y un ratón del monte, Proechimys ap.; el cuarto ejemplar fue capturado en la localidad de Aushiri, siendo también un "ratón del monte, Proechimys sp.

Los 3 primeros resultaron negativos a la presencia de Leishmania; del último roedor, Pmechimys ip., se aislé y cultivó un stock de L eishmania desde el hígado, que sometida a la caracterización por patrones isoenzimáticos, en gel de almidón, a través de 10 enzimas, resulté ser semejante a $L$. brazilienzis braziliensis. L os patrones de migración de las enzimas ASAr, ALAT, GPI, G6PD, 6PGHD, PGM Y PEP, se muestran en la Fig. 2; con un índice de similaridad de $66 \%$ (Tabla 1).

\section{DISCUSION}

Los perfiles isoenzimáticos determinados a través de la técnica de gel de almidón, permiten identificar con seguridad la categoría taxonómica de subespecie, dentro del Complejo Leishmania brasiliensis, del stock aislado del hígado del roedor Proechimys ap., desde al al to $\mathrm{N}$ apo.

La presencia de Leishmania braziliensis braziliensis en animales silvestres en esta área, es la primera comunicación de este tipo en el país.

Este hallazgo coincide con la afirmación de Lainson (13), cuando menciona que los huéspedes naturales para $\mathrm{k}$ braziliensis braziliensis son algunos roedores y que la infección en el animal es comúnmente inaparente, encontrándose los parásitos en vísceras y piel, pero no coincide cuando el mismo autor (14), menciona que el roedor del género Proechimys sp. Está infectado con L. braziliensis guyanensis y L eishmania mexicana amazonensis en la amazonía brasilera.

La cercanía entre el área de captura del roedor, quebrada Aushiri, y la residencia del paciente detectado con Leishmania braziliensis guyanensis (15), permite suponer la presencia de ambas subespecies en la misma área geográfica, lo que explicaría la casuística clínica, de formas cutáneas y cutáneomucosas encontrada (16). 
FIG. 2: PATRONES DE MIGRACION DE ISOENZIMAS

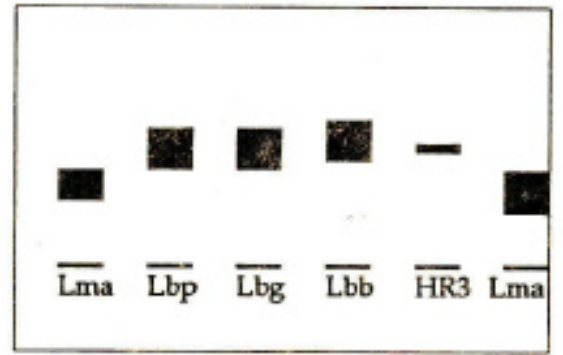

GPI

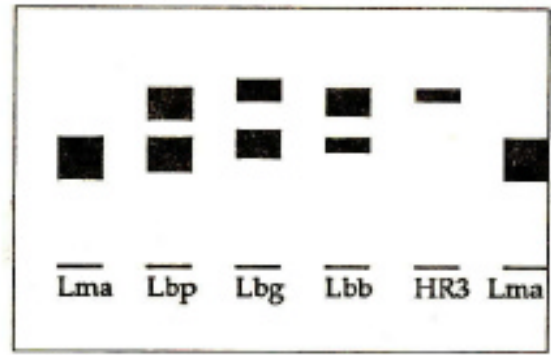

ASAT

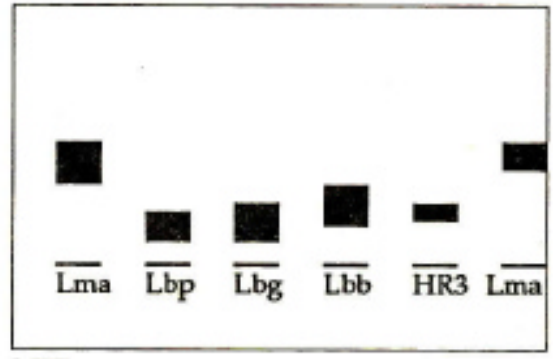

MPI

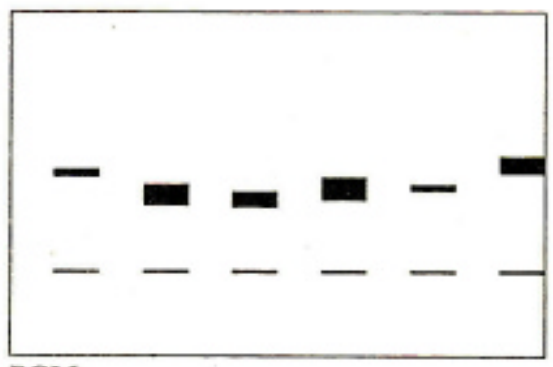

PGM
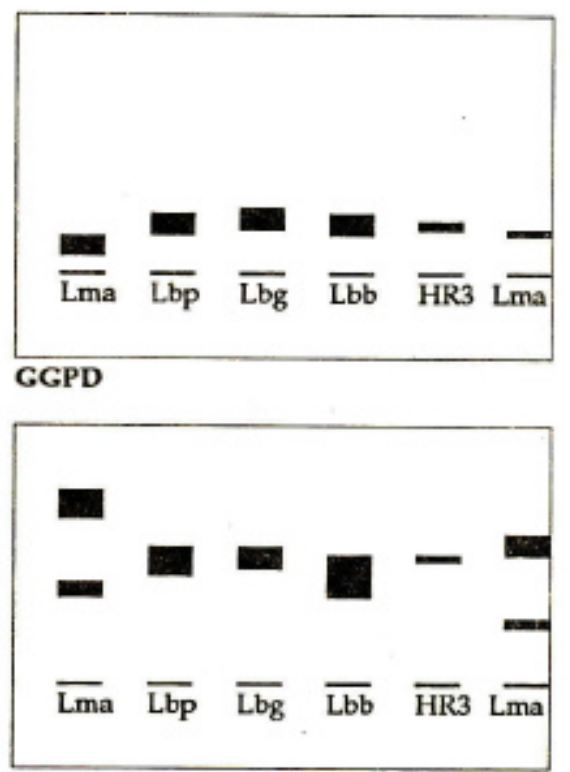

ALAT

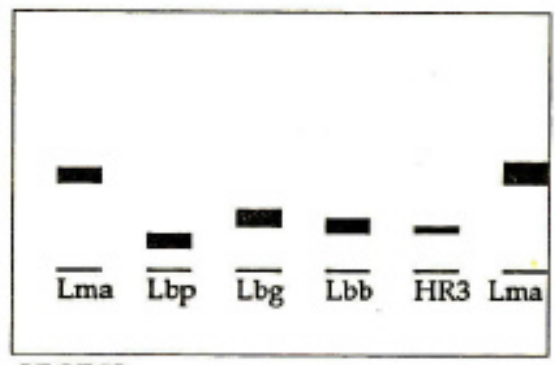

\section{GPGDH}

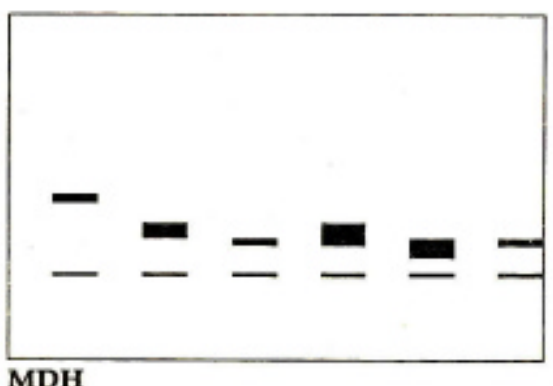



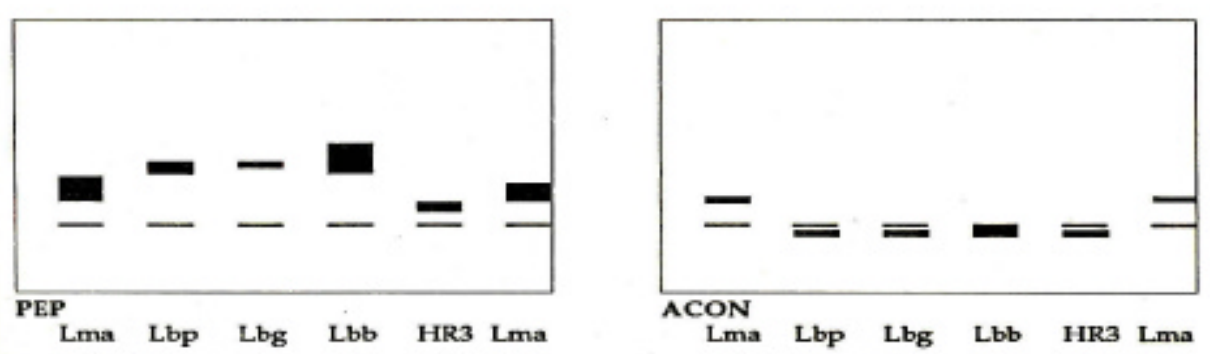

Es necesario continuar las investigaciones a este respecto, a fin de poder determinar la presencia de Leishmania en otros roedores, y la presencia de Leishmania braziliensis braziliensis en pacientes de la misma zona. L lama la atención la ubicación de Leishmania braziliensis braziliensis en las vísceras del roedor, lo que haría suponer que este tipo de Leishmania no sólo afecta la piel y mucosas, sino que produce una Leishmaniansis visceral en los roedores. Se estima que estudios posteriores arrojarán más luz sobre esta situación.

\section{BIBLIOGRAFIA}

1. Colán , E., Braga, J., García, E. Hallazgos preliminares de Leishmaniasis en Loreto. VII Congreso Latinoamericano de Parasitología. Guayaquil. Ecuador. pp.93.1985.

2. Lainson, R., Shaw J.J. Las Leishmanias y la Leishmaniasis del Nuevo M undo, con particular referencia al Brasil. Boletín de la Oficina Sanitaria Panamericana. Feb pp. 93-114. 1974

3. Lainson, R., Shaw J.J. Epidemiology and Ecology of Leishmardasis in Latino A mérica. Nature. Vol 273. J un pp. 595-600... 1978

4. Nagorsen, A.W. and Peterson, R. L. Mammals. Collector's M anual.The Royal Ontario M useum. Printed and Boun in Canadá. at the Alger Press. p. 79. - 1980.

5. Herrer, A Nature infections of Leishmania and Trypanosome demostred by skin culture. The lournal of Parasitology. V ol. 52. NI2 5. pp 954- 957-1966.

6. Cuba, C.C. Llanos Cuentas, E. A., Barreto A.C. Magalliaes, KV. Lago,. EL, Reed, S. G. and Marsden, P.O Human Mucocutaneous Leishmaniasis en Tres Braços Bahía - Brasil an area of Leishmania braziliensis braziliensis - transmission. 1. Laboratory Diagnosis. Rey, da. Soc. Bras. Med. 1ro . 17. (4): 161 -167. Out Dez. 1984. 


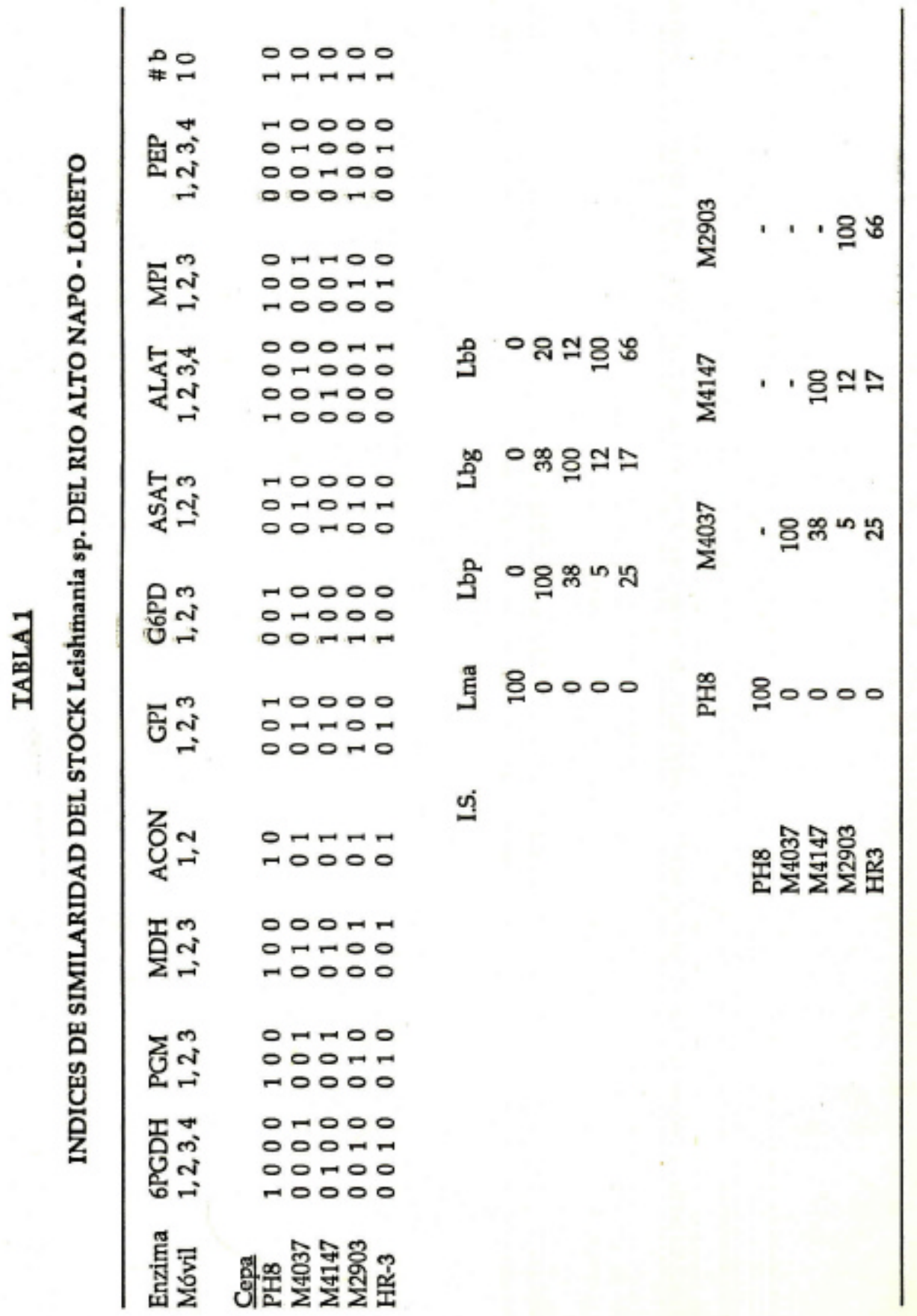


7. Hendricks, L. and W rigth, N. Diagnosis of cutaneous leishmaniasis by in viti cultivation of salme aspirates in Schneiders. Drosophila M edium.

Am.. J.Trop. Med. Hyg. 28 (6). p.962 - 964 - 1979.

8. Romero, G.G. Arana M., López, M. Montoya, I., Bohl, R., Campes,M.,J A révalo, and Llanos, $A$. Characterization of $L$ eishmania species from Peru. Trans. of the Royal . Soc. of Trop. M ed. and Hyg. 81,14 24.1987.

9. Braga, R. R., Lainson, R., Shaw, J.J., Ryan, L., and Silveira, F.T. Leishmaniasis in Brazil. XXII: Characterization of Leishmania from man, dogs, and the sandfly L ut zomya longipal pis (L utz. \& N eiva, 1912) isolated during art outbreak of viscera leishmaniasis ja Santarém, Pará,. State. Trans. of the Royal Soc. Of Trop. M ed. and Uyg. 80, 143-145.1986.

10. M iles, M.A., Linson, R., Shaw., J.J. Póvoa, M. and de Souza, A Leishmaniasis ir Brazil. XV: I3iochemical distinction of Leishmania mexicana amazonensis L eishmania braziliensis braziliensis y Leishmania braziiensis guyanensis aetiological agent of cutaneous leishmaniariaiatle A mazon. Basin of Brasil. Trans of the Royal of Trap. Med and Hyg. Vol. 75. № 4 524-529. 1981

11. Ready, P.D., and M iles. M.A. Delimitation of Trypanosome cruzi zymodemes by numerical taxonomy. Trans. of the Royal. Soc. of Trop. Med. And Hyg. Vol 74 №2 2238-242. 1979.

12. Godfrey, D. G. Biochemical characterization in the taxonomia of Parasitic. Protozoa. M. Bioch. Charact. of Leishmania. Ed. by. Chance, M. L. \& W alton, B.C. UNDP / W orld Bank/WH O. 1980.

13. Lainson, R. Epidemiología e Ecología de Leishmaniose Tegumentar na Amazonia. Heléia M edica. Belém. 3. (1): 35-40.1981

14. Lainson, R. ,Shaw, J. 1.. Ready, P. D., Miles, M. A. and Próvoa, M. Leishmaniasis in Brazil: XVI: Isolation and identiflcation of Leishmania species from sandflies, Wild niammals and man in noth .Pará State, with particular reference to. $L$. braziliensis guyanensis causative agent of "pian bois. Trans. of the Royal Soc. of Trop. Med. and Hyg. Vol. 75, pp. 530 -536. 1981.

15. Braga, J., García, E., Colán, E., Braga, R.R., A rana, M. Leishmania braziliensis brazilíensis y Leishmania braziliensis guyanensis en el $D$ epartamentos de $L$ oreto Perú 1987. (impress). 
16. Braga, J., García, E., Colán . E., Viena, M ., M arapara, J. Observaciones epidemiológicas de Leishmaniasis en el Departamento de Loreto. $X$ Congreso Latinoamericano de Microbiología y VII Congreso Peruano de Microbiología y Parasitología. Trujillo. Perú 1987. 